\title{
Rotura vesical intraperitoneal y reparación laparoscópica: ¿Es éste el nuevo gold standard?*
}

\author{
Drs. PABLO TRONCOSO C. ${ }^{1}$, ÁlVARO SAAVEDRA Z. ${ }^{1}$ MIGUEL SARRAS J. ${ }^{1}$, FRANCISCO ROJAS R. ${ }^{1}$ \\ 1 Departamento de Urología Hospital Clínico de la Pontificia Universidad Católica. \\ Santiago, Chile.
}

\begin{abstract}
Intraperitoneal bladder rupture and laparoscopic repair: is this the new gold standard?
\end{abstract}

Introduction: Nowadays laparoscopy is the new paradigm in many surgical treatments; however, its role in urological trauma is barely emerging. The aim of this article is to show a laparoscopic repair technique of an intraperitoneal bladder rupture secondary to a blunt abdominal trauma, its feasibility and safety. Case: We present a 28 year old man who consulted with 6 hours of hypogastric pain, absence of micturition and vesical globe, after alcoholic consumption. He presented high blood both creatinine and inflammatory parameters. A Foley catheter was installed, the pain increased progressively, with Blumberg sign and tachycardia. Looking back again, he told about a blow in the hypogastric region with full bladder. A non-contrasted CT of the pelvis showed the catheter's end into peritoneal cavity. A successfully three port laparoscopic repair was made using intracorporeal double layer suturing technique with poliglactin, an intraoperative cystoscopic check was performed. The patient was discharged at sixth day without complications.Discussion: Classically surgical treatment for intraperitoneal bladder rupture has been laparotomy with double layer closure of the lesion. However, there are no randomized control trials comparing this technique with other methods. Also, there is a lack of laparoscopic repair reports, probably due to most bladder ruptures are extraperitoneal (managed with non-surgical treatment) or associated with other organs injury (needing laparotomy). Although more evidence is needed, we believe that laparoscopic repair of intraperitoneal bladder rupture could be considered as an option in stable patients and without important associated injuries, achieving better cosmetic outcomes and faster recovering.

Key words: Urinary bladder, wounds injuries, laparoscopy.

\section{Resumen}

Introducción: Actualmente la laparoscopia es el nuevo paradigma en muchos tratamientos quirúrgicos, pero su rol en trauma urológico es incipiente. Nuestro objetivo es mostrar una técnica factible y segura de cistorrafia laparoscópica por ruptura vesical intraperitoneal contusa. Caso: Un hombre de 28 años consulta por 6 horas de dolor hipogástrico, retención y globo vesical, después de una transgresión alcohólica. Ingresa

*Recibido el 14 de mayo de 2012 y aceptado para publicación el 14 de julio de 2012.

Los autores no refieren conflictos de interés.

Correspondencia: Dr. Álvaro Saavedra Z.

Marcoleta 367, Santiago, Chile.

alvarosaavedraza@gmail.com 
con creatininemia y parámetros inflamatorios elevados. A pesar de un sondeo vesical exitoso, evoluciona con más dolor, Blumberg y taquicardia. Revisando la anamnesis, refiere un golpe en hipogastrio, con vejiga llena. En pieloTAC se observa el extremo de la sonda en cavidad peritoneal. En pabellón, se realiza una reparación laparoscópica mediante tres trócares, usando sutura de poliglactina en doble capa, con revisión cistoscópica intraoperatoria. El paciente se da de alta al sexto día postoperatorio, sin complicaciones. Discusión: Clásicamente, el tratamiento quirúrgico de la ruptura vesical intraperitoneal, ha sido la laparotomía exploradora con cierre de la lesión en doble capa. Sin embargo, esta técnica no ha sido comparada en estudios randomizados con otras modalidades. Además, hay escasos reportes de la técnica laparoscópica, probablemente porque la mayoría de las lesiones son extraperitoneales (con manejo conservador) o con lesión de otros órganos (requiriendo laparotomía). Se necesita más evidencia, pero creemos que la cistorrafia laparoscópica de lesiones intraperitoneales, es una buena conducta en pacientes estables sin otros daños asociados, alcanzando mejores resultados estéticos y una recuperación más rápida.

Palabras clave: Vejiga urinaria, traumatismos, laparoscopia.

\section{Introducción}

Hoy en día la laparoscopia se ha establecido como un nuevo paradigma de enfrentamiento quirúrgico. Este cambio de enfoque ha hecho plantear el tratamiento laparoscópico como el gold Standard en muchos casos, relegando a la vía abierta como una segunda opción. Sin embargo, el rol del abordaje laparoscópico en el trauma urológico no está bien definido. En el caso de la rotura vesical intraperitoneal, el Consensus statement on bladder injuries de la Société Internationale d'Urologie (SIU) ${ }^{1}$, recomienda la reparación formal por laparotomía y restringe la vía laparoscópica sólo a aquellos casos de lesión iatrogénica ocurrida en el transcurso de alguna cirugía igualmente laparoscópica. Sin embargo, estas recomendaciones son basadas en publicaciones más antiguas, cuando la cirugía mínimamente invasiva aún no alcanzaba los niveles actuales de desarrollo.

En 1994, Parra presenta la primera experiencia en reparación vesical laparoscópica ${ }^{2}$ y desde entonces sólo se han reportado casos clínicos aislados ${ }^{3-6}$ y de series pequeñas ${ }^{7,8}$.

El objetivo de esta comunicación es mostrar un caso reciente de reparación vesical laparoscópica en rotura vesical intraperitoneal por trauma cerrado y estimular la publicación de más reportes que permitan consolidar la evidencia.

\section{Caso clínico}

Paciente de sexo masculino de 28 años, sin antecedentes mórbidos, consulta en Servicio de Urgencia por un cuadro de pocas horas de evolución de dolor hipogástrico de intensidad moderada, asociado a ausencia de micción por 6-8 hrs y distensión hipogástrica dolorosa a la palpación, sugerente de un globo vesical, todo esto en relación a una transgresión alcohólica ese mismo día. Al ingreso se encontraba taquicárdico, afebril y con discretos signos de irritación peritoneal. En el Servicio de Urgencia se hace el diagnóstico de retención aguda de orina y se instala una sonda Foley que da salida a $500 \mathrm{cc}$ de orina hematúrica. Al laboratorio destacan: creatinina plasmática de $4,13 \mathrm{mg} / \mathrm{dL}$, nitrógeno ureico de 32 $\mathrm{mg} / \mathrm{dL}$, leucocitos plasmáticos de $15.300 / \mathrm{mcL}$ y PCR de 1,88 mg/L. Durante las primeras 10 horas de hospitalización el dolor hipogástrico se hace progresivo, asociando signo de Blumberg franco, taquicardia y PCR de $20,3 \mathrm{mg} / \mathrm{L}$, a pesar de una diuresis de $1.500 \mathrm{cc}$ en 12 horas. Ante el cambio en la evolución se reexplora la anamnesis en búsqueda de algún accidente, confirmándose una caída a nivel y una contusión hipogástrica con el ángulo de una escalera, aparentemente estando la vejiga llena. Se solicita un TC de abdomen y pelvis sin contraste (por precaución ante la elevada creatinina plasmática) que muestra la sonda Foley con su extremo en la cavidad peritoneal, leve ascitis y abundantes coágulos en vejiga (Figura 1). Se decide ingresar a pabellón con el diagnóstico de una ruptura vesical intraperitoneal.

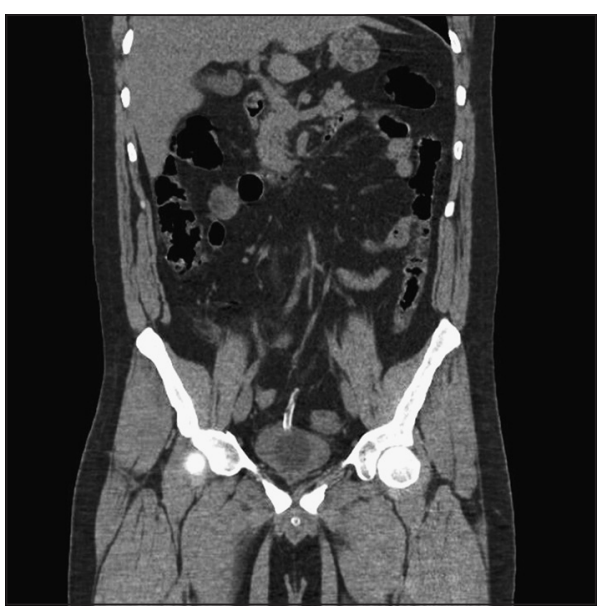

Figura 1. Sonda Foley con cuff en vejiga y extremo en fondo de saco peritoneal. 


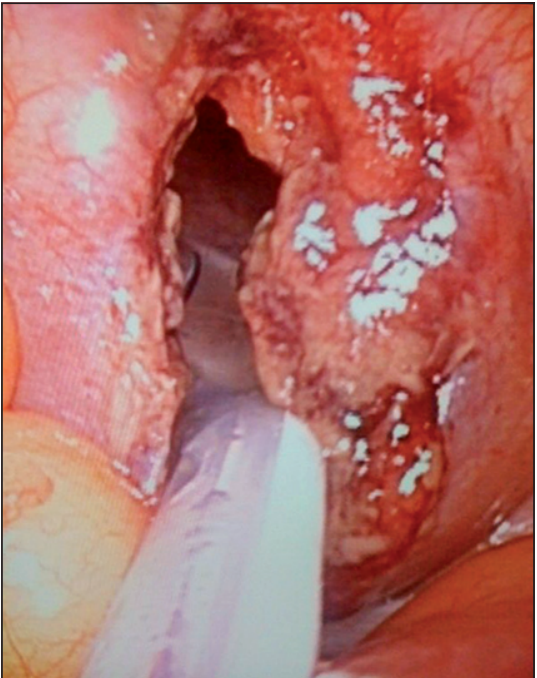

Figura 2. Vista laparoscópica de la lesión vesical con la sonda Foley in situ, llegando al fondo de saco.

\section{Técnica quirúrgica}

a. Posición. Decúbito supino, en Trendelemburg leve.

b. Trócares y pneumoperitoneo. Se instala primer trocar con técnica de Hassan, en línea media, inmediatamente subumbilical. Se introduce un trocar de $5 \mathrm{~mm}$ en fosa ilíaca izquierda y otro de $10 \mathrm{~mm}$ en fosa ilíaca derecha, ambos bajo visión y transiluminación directa, en línea axilar media. El paciente ingresa a pabellón con la sonda previamente instalada y se clampea para evitar fuga de pneumoperitoneo. Se insufla abdomen con una presión de $15 \mathrm{mmHg}$.

c. Hallazgos. Con óptica de $0^{\circ}$ se observa escasa cantidad de líquido libre en el fondo de saco y una ruptura en forma estrellada en la cúpula vesical, de unos $3,5 \mathrm{~cm}$, con el extremo de la sonda vesical insinuada en la cavidad peritoneal (Figura 2).

d. Reparación. Se libera el plano peritoneal de la pared vesical y luego de corroborar la viabilidad de los tejidos, se procede a cerrar mucosa y detrusor en un mismo plano con sutura continua de poliglactina 1-0. Luego se hace cierre del peritoneo con sutura continua de poliglactina 2-0. Posteriormente se verifica la hermeticidad con cistoscopia simultánea, que no muestra lesiones adicionales, ni tampoco evidencia de filtraciones (Figura 3).

e. Cierre. Se instala un drenaje tubular en el fondo de saco, a través de uno de los trócares, dejándose a caída libre. Se instala sonda Foley siliconada de 20 Fr y luego se cierra la incisión del trocar de

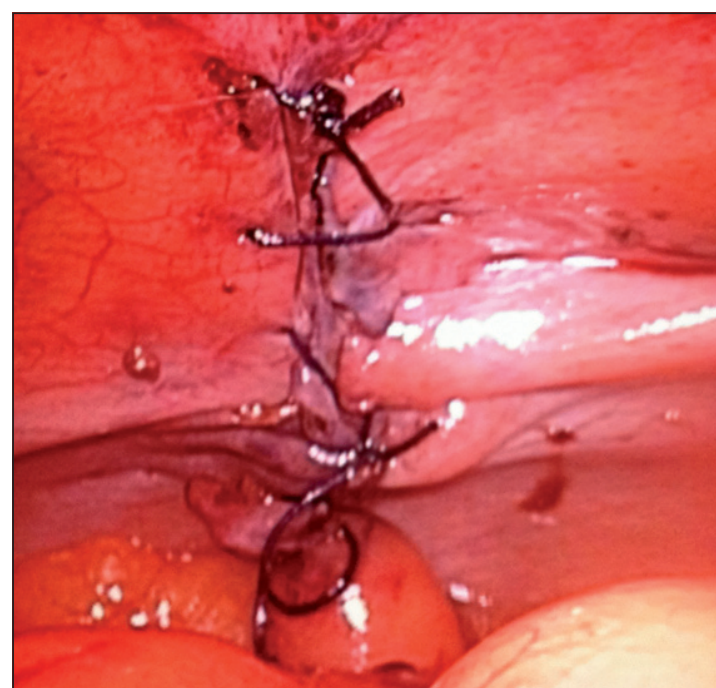

Figura 3. Vista final de la reparación en dos planos (detrusor y peritoneo).

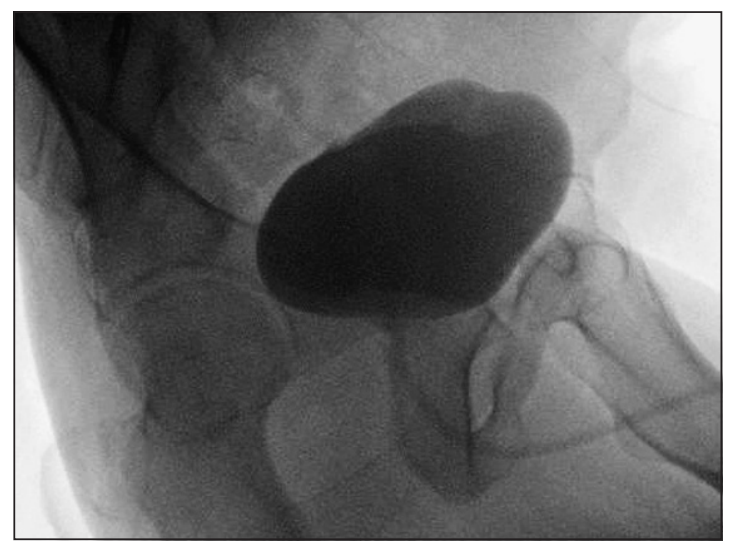

Figura 4. Uretrocistografía al $13^{\circ}$ día postoperatorio, en llene forzado, que no muestra filtraciones.

Hassan con puntos separados de Vicryl 1-0. Cierre de piel con sutura intradérmica con Monocryl 3-0 en todas las incisiones.

\section{Postoperatorio}

El paciente evoluciona favorablemente, afebril, requiriendo bajas dosis de analgesia endovenosa, que se suspenden al segundo día. Tolera régimen oral a las 12 horas de operado. Con buena diuresis por sonda, la creatinina plasmática baja a $1,07 \mathrm{mg} / \mathrm{dL}$ a las $24 \mathrm{hrs}$ postoperatorias y a $0,88 \mathrm{mg} / \mathrm{dL}$, previo al alta. Se da alta con sonda Foley al sexto día. A los 13 días es evaluado con cistografía que no muestra filtraciones (Figura 4), y la sonda es retirada a los 16 días del postoperatorio. 


\section{Discusión}

Hasta el momento, el enfrentamiento clásico de la ruptura vesical intraperitoneal ha sido la laparotomía y cierre de la lesión en dos planos, con instalación de drenaje peritoneal y ocasionalmente el uso de cistostomía. La ausencia de estudios randomizados que comparen la técnica abierta con otros métodos, ha estimulado el desarrollo de nuevos abordajes. Lamentablemente, el número de publicaciones que reportan reparaciones laparoscópicas es escaso, probablemente por dos razones: un número importante de los casos se asocia a otras lesiones (lesión de recto, fractura de pelvis, trauma encefálico, entre otras), lo que generalmente desincentiva la exploración mínimamente invasiva; por otro lado, el $55 \%$ de las rupturas vesicales es extraperitoneal ${ }^{1}$, requiriendo sólo manejo conservador con sonda. Si consideramos la poca casuística y que las recomendaciones no han sido actualizadas, es esperable que sigamos teniendo limitados reportes de la cirugía laparoscópica.

En este caso clínico, es relevante considerar que en un comienzo el paciente no refirió el episodio de trauma, por su estado de ebriedad ${ }^{5}$, lo que sólo se logró con un interrogatorio muy dirigido. La elevación importante y sin explicación clara de la creatininemia, debe sugerir la lesión intraperitoneal y uroperitoneo ${ }^{1}$, sin contraindicar necesariamente el uso de estudios contrastados. En este paciente, la sonda vesical permitió evidenciar la ruptura aún en un estudio sin contraste, que de otro modo sólo hubiera mostrado líquido libre.

Parece obvia la suma importancia de contar con imágenes del abdomen y la pelvis, pues permiten evaluar el estado de los órganos adyacentes, estimar la extensión de la lesión, descartar otros diagnósticos y planificar la vía de abordaje. Se recomienda también el uso de uretrocistografía o cistoscopia intraoperatorias, para corroborar la calidad de la reparación ${ }^{7}$.

En general, todos los reportes de casos únicos han utilizado técnicas similares: tres trocares, sutura en dos capas y drenaje, mostrando buenos resultados. Cabe destacar la utilidad del laparoscopio, pues permite ingresar a la vejiga a través de la misma lesión y explorarla con magnificación, requiriéndose el clampeo de la sonda vesical, para así mantener la presión del pneumoperitoneo ${ }^{5}$. Esto evita la necesidad de extender la incisión y la cistotomía para lograr la exposición, situación frecuente en la laparotomía.

$\mathrm{Kim}$ et $\mathrm{al}^{7}$, presentan la primera serie de 6 casos de reparación laparoscópica $(5,4 \%$ del total de reparaciones hechas en 4 años). Ellos usaron sutura en monocapa y drenaje, con buenos resultados, aplicando los siguientes criterios de inclusión: (a) estabilidad hemodinámica, (b) ausencia de daño en otros órganos intraabdominales y (c) ausencia de lesión craneal. El tiempo quirúrgico promedio fue de 43 minutos y no tuvieron complicaciones intraoperatorias ni postoperatorias.

En el postoperatorio es imprescindible evaluar el estado de la reparación, previo al retiro de la sonda vesical, con una cistografía en dos tiempos: en llene forzado y en vaciado ${ }^{1}$. Este último tiempo permite confirmar la indemnidad del cuello.

En los reportes publicados, este tipo de abordaje ha demostrado ser seguro, eficaz, con pocas complicaciones y asociado a los beneficios estéticos propios de la técnica ${ }^{2-9}$. En los infrecuentes casos de lesiones extraperitoneales concomitantes, el sondeo vesical postoperatorio constituye el tratamiento de elección ${ }^{1}$.

Aunque se requiere de mayor evidencia y de estudios comparativos que permitan actualizar las recomendaciones del manejo de la ruptura vesical intraperitoneal, hasta el momento, el abordaje laparoscópico puede considerarse como una opción factible en pacientes estables y sin lesiones asociadas importantes. Este enfoque permite mejores resultados estéticos, menos requerimientos de analgesia y, probablemente, una reinserción laboral más precoz. Nuestro trabajo pretende estimular el uso de esta técnica quirúrgica en pacientes seleccionados y fomentar el reporte de casos y series, fortaleciendo la evidencia que permita incluir su uso en las guías clínicas futuras.

\section{Referencias}

1. Gómez R, Ceballos L, Coburn M, Corriere J, Dixon C, Lobel B, et al. Consensus statement on bladder injuries. BJU Int. 2004;94:27-32.

2. Parra RO. Laparoscopic repair of intraperitoneal bladder perforation. J Urol. 1994;151:1003-5.

3. Mendoza-Lucio LA, García-Morúa A, Gómez-Guerra L, Sepúlveda-Malec R, Martínez-Montelongo R. Ruptura vesical traumática manejada exitosamente por cirugía laparoscópica. Rev Mex Urol. 2008;68:289-91.

4. Figueiredo A, Tostes J, Jacob M. Laparoscopic Treatment of Traumatic Intraperitoneal Bladder Rupture. Int Braz J Urol. 2007;33:380-2.

5. Appeltans BM, Schapmans S, Willemsen PJA, Verbruggen PJ, Denis LJ. Urinary bladder rupture: Laparoscopic repair. Br J Urol. 1998;81:764-5.

6. Matsui Y, Ohara H, Ichioka K, Terada N, Yoshimura K, Terai A. Traumatic bladder rupture managed successfully by laparoscopic surgery. Int J Urol. 2003;10:278-80.

7. Kim F, Chammas M, Varella E, Campagna A, Moore E. Laparoscopic management of intraperitoneal bladder 
rupture secondary to blunt abdominal trauma using intracorporeal single layer suturing technique. J Trauma. 2008;65:234-6.

8. Duque M, Ceballos M, Plaza L, Mendoza A, García D. Reparo laparoscópico de la ruptura vesical intraperito- neal de origen traumático. ¡El nuevo patrón de oro! Urol Colomb. 2010;19:73-6.

9. Tay KP, Ravintharan T, Lim PHC, Chang HC. Emergency laparoscopic repair of spontaneous and traumatic bladder rupture. Br J Urol. 1995;75:550-1. 